\title{
Down syndrome and recent demographic trends in Manitoba
}

\author{
JANE A. EVANS, A. G. W. HUNTER, AND J. L. HAMERTON \\ From the Division of Genetics, Department of Paediatrics, University of Manitoba and Health Sciences Centre, \\ Winnipeg, Manitoba, Canada
}

SUMmaRY Two hundred and thirty-three children born in Manitoba with Down syndrome between 1965 and 1974 were ascertained and the maternal ages obtained. Mean maternal age was found to have declined in this period both for all livebirths and to a greater extent for Down syndrome children. Though the proportion of mothers of Down syndrome infants with a maternal age of less than 35 years remained the same, the proportion of mothers aged 35 to 39 years increased and the age specific incidence of Down syndrome became significantly greater for women in this age group. Reduction in the proportion of Down syndrome births to women over 40 years and the increased incidence of Down syndrome in children of women aged 35 to 39 years has important consequences for the planning of amniocentesis programmes.

Recently much attention has been focused in several countries on the possible influence of the decline in mean maternal age on the incidence of Down syndrome (Uchida, 1970; Gardner et al., 1973; Lindsjö, 1974; Lowry et al., 1976; Mikkelsen et al., 1976).

Mean maternal age and total number of livebirths have been decreasing in Manitoba since 1962. This reduction in maternal age should have led to a lower incidence of Down syndrome because of the wellknown association of Down syndrome with increased maternal age. Uchida (1970) investigated Down syndrome in Manitoba from 1960 to 1968 . This study revises the data on Down syndrome in Manitoba from 1965 to 1968 and presents new information on the incidence of Down syndrome from 1969 to 1974 with special reference to maternal age.

\section{Methods}

Two hundred and thirty-six patients with Down syndrome born between 1965 and 1974 were ascertained primarily through the Provincial Registry for Congenital Anomalies and records of the Genetic Department of the Children's Centre, Winnipeg. Other sources contributed less than $2 \%$ of new index patients. Where reference is made to the period 1960 to 1964, Uchida's data (Uchida, 1970) were used.

Received for publication 13 June 1977
The age of the mothers at the birth of each affected child was obtained from the Provincial Registry for Congenital Anomalies; the Manitoba Health Services Commission, the hospital of birth, or Vital Statistics.

\section{Results}

Three of the 236 index cases were born outside Manitoba and were excluded from further analysis. Chromosome studies were available on $184(79 \%)$ of children and are summarised in Table 1 . The remaining 49 cases were clinical diagnoses. Twenty-eight cases born between 1965 and 1968 were not ascertained by Uchida's study (Uchida, 1970). Further

Table 1 Cytological analysis of Down syndrome children

\begin{tabular}{lr}
\hline Standard G trisomy & No. \\
$47, X Y$ or $X X,+G$ & 174 \\
$47, X Y$ inv $19(\mathrm{p} 13 ; q 13),+21$ & 1 \\
$46, X,+G / 47, X Y,+G$ & 1 \\
Total & 176 \\
Translocation Down syndrome & \\
$46, X Y,-G, t(G q G q)$ & 2 \\
$46, X X,-G, t(G q G q)$ & 1 \\
$46, X Y,-D, t(D q G q)$ & 1 \\
$46, X X,-D, t(D q G q)$ & 1 \\
Total & 5 \\
Mosaic Down syndrome & \\
$46, X Y / 47, X Y,+21$ & 2 \\
$46, X X / 47, X X,+21$ & 1 \\
Total & 3 \\
\hline
\end{tabular}


studies were carried out only on the sample of children with standard trisomy 21 .

There was a non-significant excess of male children (130:105). An additional child had ambiguous genitalia but died before karyotypic sex was determined. There were 6 twin pairs of unconfirmed zygosity. Two female sets were concordant for Down syndrome. A macerated female fetus was found 'tucked in' to the single placenta of another affected girl. Of 3 affected boys, one had a normal male twin, one a normal female twin, and the sex of one normal co-twin was unknown. Four families had 2 affected children. In 2 families both children were confirmed as having standard trisomy 21 . In the other 2 families, one child had standard trisomy 21 while the other was not examined cytogenetically.

MATERNAL AGE

The number of livebirths in Manitoba has fallen from 23237 in 1960 to 17609 in 1974 . There has been a drop in age specific fertility in all age groups but especially among older women. Between 1960 and 1974 the percentage of babies born to women 35 and over fell from $14.5 \%$ to $5 \%$ (Table 2). This change in maternal age distribution should have led to a fall in the number of children born with Down syndrome if the age specific incidence of Down syndrome has remained unchanged.

In Manitoba the mean maternal age has fallen from 27.2 years in 1960 to 25.1 years in 1974. In the same period the mean maternal age for Down syndrome births fell from 34.8 years to 30.6 years. The mean maternal age for Down syndrome birth (Fig. 1) shows considerable fluctuation from year to year and the regression of maternal age on time based on yearly data is not significant. When the data are pooled in 2-year age ranks (3 years for 1972 to 1974) regression of maternal age on time is significant $(\mathrm{P}<$ $0.025)$.

In 1967 there was a significant reduction in mean maternal age compared to the regression line of mean maternal age on time for 1960-1974 (P<0.02). In this year 76 per cent of mothers of Down syndrome

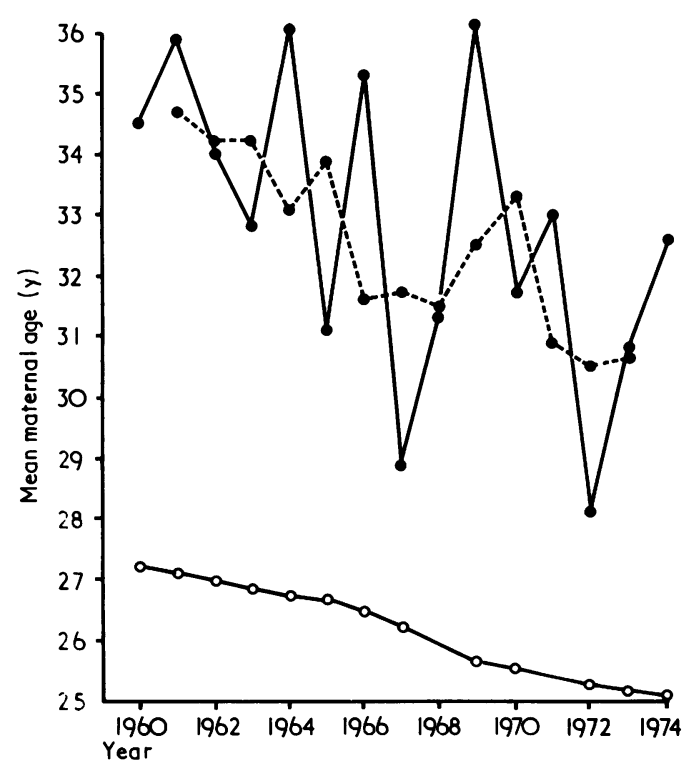

Fig. 1 Mean maternal age by year for 1960 to 1974 Down syndrome infants individual years, Down syndrome infants 3-year moving averages, $\mathrm{O}-\mathrm{O}$ all livebirths).

infants were under 35 years. The cause of this reduction is not known.

It is possible that a cyclical component does exist in these data and that mean maternal age fluctuates in 3 to 4 year cycles. Using the technique of moving averages (for example, the mean of the first 3 years, the mean of years 2 to 4 , years 3 to 5 , etc., gives a 3 year moving average series), it is possible to remove the cyclical component from a set of data and attempt to isolate it (Floud, 1973). Regression of 3-year moving averages of mean maternal age on time (Fig. $1)$ is highly significant $(P<0.001)$. However, whether this is a real periodicity or is spurious remains to be seen, and until a predisposing factor for Down syndrome that fluctuates with this periodicity can be

Table 2 Major demographic trends in Manitoba 1965 to 1974

\begin{tabular}{|c|c|c|c|c|c|c|c|c|}
\hline \multirow{4}{*}{$\begin{array}{l}\text { Year } \\
1965 \\
1966\end{array}$} & \multirow{3}{*}{$\begin{array}{l}\text { No of } \\
\text { Down births }\end{array}$} & \multirow{3}{*}{$\begin{array}{l}\begin{array}{l}\text { No. of } \\
\text { livebirths }\end{array} \\
19976\end{array}$} & \multicolumn{3}{|c|}{ \% livebirth by maternal age } & \multicolumn{3}{|c|}{ Age specific fertility } \\
\hline & & & $10-34$ & $35-39$ & $40-49$ & \multicolumn{3}{|c|}{ (expressed as livebirths/1000 women) } \\
\hline & & & 86.4 & 9.9 & 3.7 & $98 \cdot 2$ & 67.4 & $12 \cdot 5$ \\
\hline & 23 & 18007 & 87.0 & $9 \cdot 3$ & $3 \cdot 7$ & $87 \cdot 3$ & $59 \cdot 0$ & $11 \cdot 5$ \\
\hline 1967 & 25 & 17180 & $88 \cdot 2$ & $8 \cdot 7$ & $3 \cdot 1$ & 83.4 & 53.4 & $9 \cdot 2$ \\
\hline 1968 & 31 & 17424 & 89.7 & 7.6 & $2 \cdot 7$ & $83 \cdot 8$ & $49 \cdot 0$ & $8 \cdot 0$ \\
\hline 1969 & 14 & 17809 & $90 \cdot 1$ & $7 \cdot 3$ & $2 \cdot 6$ & $83 \cdot 8$ & $49 \cdot 0$ & $8 \cdot 1$ \\
\hline 1970 & 25 & 18248 & 91.7 & $6 \cdot 2$ & $2 \cdot 0$ & $85 \cdot 8$ & 44.4 & $6 \cdot 5$ \\
\hline 1971 & 19 & 18031 & $92 \cdot 2$ & 5.9 & 1.9 & 83.5 & $41 \cdot 5$ & $6 \cdot 1$ \\
\hline 1972 & 22 & 17398 & $93 \cdot 2$ & $5 \cdot 2$ & $1 \cdot 6$ & $80 \cdot 0$ & $35 \cdot 9$ & $5 \cdot 0$ \\
\hline 1973 & 13 & 16964 & $94 \cdot 1$ & $4 \cdot 5$ & 1.4 & $77 \cdot 0$ & 30.4 & $4 \cdot 5$ \\
\hline 1974 & 25 & 17609 & 95.0 & 3.9 & $1 \cdot 1$ & $74 \cdot 1$ & $25 \cdot 9$ & 3.6 \\
\hline
\end{tabular}


recognised, the data may be assumed to reflect a longterm trend with irregular fluctuation.

\section{Discussion}

\section{MATERNAL AGE DISTRIBUTION}

This investigation has shown that the mean maternal age of Down syndrome infants has fallen in Manitoba during the last decade. When regressions of mean Maternal age on time for all live births and for Down syndrome births are compared, there is a significant difference $(P<0.001)$. Maternal age for Down syndrome births has fallen at a significantly greater rate $(0.29$ years/year $)$ than maternal age for all livebirths $(0.16$ years/year). This is to be expected as $45 \%$ of mothers of Down syndrome children were 35 years or older compared with 5 to $15 \%$ of mothers of all liveborn children. Reduction in fertility in older mothers would, therefore, cause a proportionately greater decline in mean maternal age in the Down syndrome group.

Uchida (1970) reported a drop in mean maternal age from 27.2 years to 26.2 years from 1960 to 1967 . However, in her sample of Down syndrome children, mean maternal age did not show a decline but fluctuated around 34 years with a sudden decrease to 29.3 years in 1967. There was no significant change in the total incidence in this period. The decline in mean maternal age in Down syndrome in Manitoba is, therefore, a relatively recent phenomenon.

Such a decline has been noted in other populations. In British Columbia mean maternal age of all livebirths has dropped from 27.4 years in 1952 to 25.0 years in 1975 (0.11 years/year) (Lowry et al., 1976). Maternal age for Down syndrome births declined from 34.1 years to 28.8 years during the same period $(0.24$ years/year). The decline in mean maternal age in Down syndrome births was more distinct from 1965 to $1973(0.36$ years/year). In Japan, the overall rate of decline in mean maternal age of Down syndrome births was 0.29 years/year from 1944 to 1963 and 0.35 years/year from 1964 to 1972 (Shiono et al., 1975). These studies show a greater rate of decline in mean maternal age than noted in Manitoba, though both from Uchida's findings (Uchida, 1970) and our own data, a similar pattern is apparent. An earlier study in Australia (Collman and Stoller, 1962) did now show any decline in mean maternal age of Down syndrome infants from 1942 to 1957 despite a reduction in mean maternal age for all livebirths, and again suggests that declining mean maternal age in Down syndrome may be a relatively recent trend.

The bimodal nature of the age rank distribution of maternal age in Down syndrome has long been recognised (Penrose and Smith, 1966), and it is apparent in most of the studies referred to, including our own. However, it is also apparent that maternal age distribution in Down syndrome is shifting both in Manitoba and elsewhere. In Manitoba, the proportion of all Down syndrome infants born to women 35 years and older fluctuated around $45 \%$ from 1965 to 1974 and there was no significant decline in this figure $(P>0.5)$. However, the percentage of Down syndrome children born to women aged 35 to 39 years increased from a mean of $16 \%$ in 1965 to 1969 to a mean of $26 \%$ in 1970 to $1974(P<0.01)$ while the percentage born to women 40 years and older decreased from a mean of $29 \%$ in 1965 to 1969 to a mean of $15 \%$ in 1970 to $1974(P<0.01)$ (Fig. 2). This is, in part, because of a lower birth rate in mothers 40 years and older but real changes in incidence are apparent (vide infra).

In British Columbia, the proportion of mothers of Down syndrome infants under 35 years rose from $46 \%$ in the period 1952 to 1955 to $80 \%$ in 1972 to 1973 (Lowry et al., 1976). In Japan the proportion of Down syndrome births to mothers 30 years and older has been declining since 1959 to 1963. However, this decline, while apparent in the 30 to 34 year cohort and in the over 40 age group has not been seen in the 35 to 39 year rank which continues to have $15 \%$ of the total Down syndrome births (Shiono et al., 1975). In Manitoba, future years may see a further increase in Down syndrome to younger mothers and a further downward shift in maternal age distribution for these births. Continued analysis of data from 1975 onwards will elucidate this. At the present time, complete ascertainment of all Down syndrome births in Manitoba in 1975 and 1976 has not been made. However, for the 13 known Down syndrome births in 1975 , the mean maternal age was 28.0 years and $31 \%$

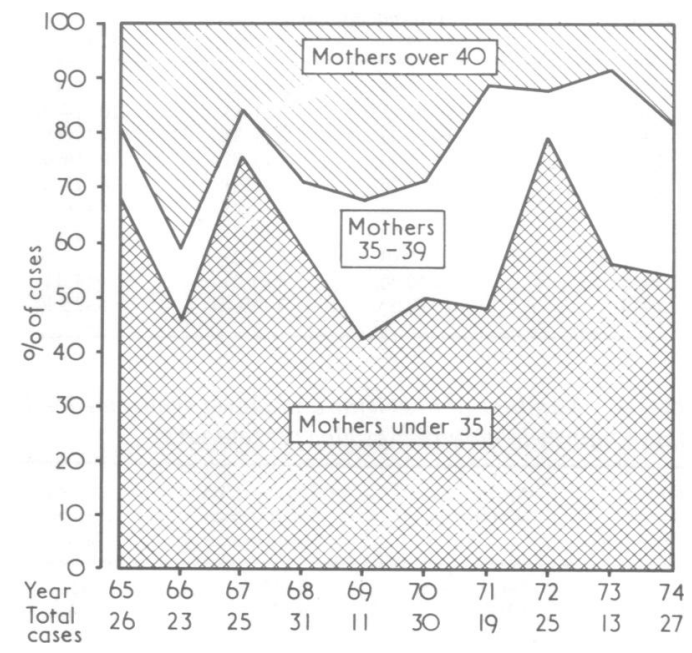

Fig. 2 Percentages of Down syndrome births to mothers under 35 years, 35-39 years, and over 40 years for 1965 to 1974. 
of these births were to mothers over 35 years. For the 14 known Down syndrome births from 1976, these figures were 27.4 years and $14 \%$, respectively. This suggests that the downward shift in mean maternal age is definitely continuing.

\section{INCIDENCE RATES}

In Manitoba the incidence of Down syndrome in mothers under 35 years $(0 \cdot 80 / 1000$ livebirths $)$ has shown no significant change from 1965 to 1974 (P > $0.5)$. In women aged 35 to 40 years, however, the regression of incidence rates per year on time shows a significant increase $(P<0.025)$. The mean incidence for this group was $2.44 / 1000$ livebirths from 1965 to 1969 and 5.69/1000 livebirths from 1970 to 1974 . There has been an increase in absolute numbers of Down syndrome births to mothers aged 35 to 39 years from 19 in 1965 to 1969 to 26 in 1970 to 1974 despite the falling numbers of livebirths to mothers in this age group $\left(\chi^{2}=8.37 \mathrm{P}<0.005\right)$ (Table 3$)$. It is highly improbable that the changes in absolute numbers and incidence of Down syndrome births to mothers in this age group is caused by chance or by small sample size.

In women 40 years and older the numbers of Down syndrome births has fallen from 33 in 1965 to 1969 to 17 in 1970 to 1974 . However, the incidence rates have not altered significantly $(P>0.25)$ from 1965 to 1969 (11.50/1000 livebirths) to 1970 to $1974(11.96 / 1000$ livebirths) though fluctuations from year to year are observed.

Table 3 Numbers of Down syndrome births (figures in parentheses give incidence/1000 livebirths)

\begin{tabular}{|c|c|c|c|}
\hline \multirow[t]{2}{*}{ Year } & \multicolumn{3}{|c|}{ Maternal age ranks } \\
\hline & $10-34$ & $35-39$ & 40.49 \\
\hline 1965 & $18(1.0)$ & $6(3 \cdot 0)$ & $4(5.4)$ \\
\hline 1966 & $10(0.6)$ & $3(1.8)$ & $10(14.9)$ \\
\hline 1967 & $19(1 \cdot 3)$ & $2(1 \cdot 3)$ & $4(7 \cdot 5)$ \\
\hline 1968 & $17(1 \cdot 1)$ & $5(3.8)$ & $9(19.4)$ \\
\hline 1969 & $5(0.3)$ & $3(2 \cdot 3)$ & $6(12.8)$ \\
\hline 1970 & $14(0.8)$ & $5(4.4)$ & $6(16 \cdot 1)$ \\
\hline 1971 & $9(0.5)$ & $8(7 \cdot 5)$ & $2(5.9)$ \\
\hline 1972 & $17(1.0)$ & $2(2 \cdot 2)$ & $3(11 \cdot 0)$ \\
\hline 1973 & $8(0.5)$ & $4(5 \cdot 3)$ & $1(4 \cdot 1)$ \\
\hline 1974 & $13(0.8)$ & $7(10 \cdot 2)$ & $5(25 \cdot 8)$ \\
\hline
\end{tabular}

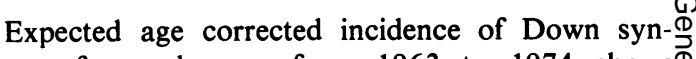
drome for each year from 1963 to 1974 shows significant decrease with time $(P<0.001)$ and if the observed regression of incidence with time is com- $\stackrel{\vec{P}}{\rightarrow}$ pared with the expected incidence regression, analysis of variance shows no significant variation $(P>0.5)$.믐 This suggests that most of the apparent overalls decrease in incidence in Down syndrome in Manitoba $\mathbb{Q}$ can be explained by changes in maternal age distri-n bution and age specific fertility. However, this will not explain the increase in the 35 to 39 year group.Neither can this increase be explained by assuming $\vec{\omega}$ mothers who would have had children in their fortieso are now completing their families at 38 or 39 years of age. When the 35 to 39 year age group is broken dowro into single years, the main increase in incidence haser been to women aged 36 and 37 , while the other years all show smaller increases. Despite the overall reduc $\vec{w}$ tion in total births in women aged 35 to 39 years, theo distribution of births between the individual year ranks 35 to 39 has remained almost static from 1965 to 1974 (Table 4). Mikkelsen et al. (1976) ino Copenhagen also noted an increase in incidence of Down syndrome in certain age ranks, notably 35 to 38 and 20 to 24 years.

If, as has been postulated (Uchida et al., $1968 \stackrel{0}{0}$ Lilienfeld and Benesch, 1969; Alberman et al., 1972); increased nondisjunction is associated with the cumulative effects of exposure to environmental agents such as radiation or chemicals, it will be interesting tō determine whether the areas showing the most rapiक̋ decline in mean maternal age and increase in age specific incidence for young mothers of Down syn $\overrightarrow{\overrightarrow{0}}$ drome infants also have higher levels of exposure to such environmental factors. Clearly environmentab agents such as diagnostic $x$-rays, nuclear fallout? exposure to pesticides, and other environmentas chemicals have become more significant in recent years and women might be expected to be exposed to threshold doses of these factors at an increasingly. early age.

The reduction in mean maternal age for Dow syndrome has important consequences for genetio counselling and prenatal diagnosis. In Manitoba, less than $15 \%$ of Down syndrome cases will be ascertaine

Table 4 Numbers of livebirths and Down syndrome births and Down syndrome incidence for maternal ages 35 to 39 years (figures in parentheses give percentages)

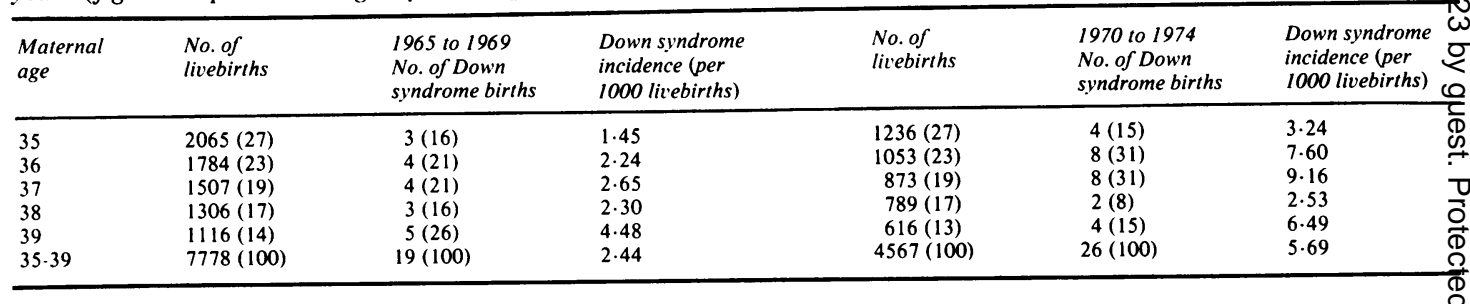


prenatally by the provision of amniocentesis to all pregnant women 40 years of age and older. The increase in incidence of Down syndrome in 35- to 39year-old women is disturbing and provision of amniocentesis to all expectant mothers in this age group would detect a further $30 \%$ of cases. Differences in maternal age distribution and age specific fertility between populations obviously necessitate the tailoring of amniocentesis programmes to individual areas.

We would like to thank Dr J. Eadie and Mrs Margaret Chauvier of the Provincial Registry of Congenital Anomalies, Manitoba, for the provision of data on many cases on Down syndrome previously unknown to our department. Their assistance in obtaining information on place and dates of birth and maternal ages was also very valuable. Thanks must also go to the medical librarians at the St Amant Centre, Winnipeg and the Manitoba School, Portage la Prairie, who also provided details of their relevant patients. The co-operation of hospital record technicians throughout Manitoba who supplied most of the maternal age data is also gratefully acknowledged.

\section{References}

Alberman, E., Polani, P. E., Fraser Roberts, J. A., Spicer, C. C., Elliott, M., and Armstrong, E. (1972). Parental exposure to Xirradiation and Down's syndrome. Annals of Human Genetics, 36, 195-208.
Collman, R. D., and Stoller, A. (1962). A survey of mongoloid births in Victoria, Australia, 1942-1957. American Journal of Public Health, 52, 813-829.

Floud, R. (1973). An Introduction to Quantitative Methods for Historians. Methuen, London.

Gardner, R. J. M., Veale, A. M. O., Parslow, M. I., Becroft, D. M. O., Shaw, R. L., Fitzgerald, P. H,Hutchings, H. E., McCreanor, H. R., Wong, J., Eiby, J. R., Howard, D. A., and Whyte, S. E. (1973). A survey of 972-cytogenetically examined cases of Down's syndrome. New Zealand Medical Journal, 78, 403-409.

Lilienfeld, A. M., and Benesch, C. H. (1969). Epidemiology of Mongolism. Johns Hopkins Press, Baltimore.

Lindsjö, A. (1974). Down's syndrome in Sweden. Acta Paediatrica Scandinavica, 63, 571-576.

Lowry, R. B., Jones, D. C., Renwock, D. H. G., and Trimble, B. K. (1976). Down syndrome in British Columba, 1952-73. Incidence and mean maternal age. Teratology, 14, 29-34.

Mikkelsen, M., Fischer, G., Stene, J., Stene, E., and Petrson, E. (1976). Incidence study of Down's syndrome in Copenhagen, 1960-71: with chromosome investigation. Annals of Human Genetics, 40, 177-182.

Penrose, L. S., and Smith, G. F. (1966). Down's Anomaly. Churchill, London.

Shiono, H., Kadowski, J., and Nakao, T. (1975). Maternal age and Down's syndrome. The shift of affected infants to younger mothers in Hokkaido. Clinical Pediatrics, 14, 241 - 244.

Uchida, I. A. (1970). Epidemiology of mongolism; the Manitoba study. Annals of the New York Academy of Sciences, 171, 361369.

Uchida, I. A., Holunga, R., and Lawler, C. (1968). Maternal radiation and chromosomal aberrations. Lancet, 2, 1045-1049.

Requests for reprints to Dr Jane A. Evans, Department of Genetics, Health Sciences Children's Centre, 700 William Avenue, Winnipeg, Manitoba, Canada R3E OW1. 